Purdue University

Purdue e-Pubs

2018

\title{
A Point Sink Superposition Method for Predicting Droplet Interaction Effects During Vapor-Diffusion-Driven Dropwise Condensation in Humid Air
}

J. E. Castillo

Purdue University

J. A. Weibel

Purdue University, jaweibel@purdue.edu

Follow this and additional works at: https://docs.lib.purdue.edu/coolingpubs

Castillo, J. E. and Weibel, J. A., "A Point Sink Superposition Method for Predicting Droplet Interaction Effects During Vapor-Diffusion-Driven Dropwise Condensation in Humid Air" (2018). CTRC Research Publications. Paper 360.

http://dx.doi.org/https://doi.org/10.1016/j.ijheatmasstransfer.2017.11.045

This document has been made available through Purdue e-Pubs, a service of the Purdue University Libraries.

Please contact epubs@purdue.edu for additional information. 


\title{
A point sink superposition method for predicting droplet interaction effects during vapor-diffusion-driven dropwise condensation in humid air
}

\author{
Julian E. Castillo and Justin A. Weibel
}

\author{
School of Mechanical Engineering, Purdue University, West Lafayette IN 47907 USA. \\ ${ }^{*}$ Corresponding author. \\ E-mail addresses: castil19@ @urdue.edu (Julian E. Castillo), jaweibel@ purdue.edu (Justin A. Weibel)
}

\begin{abstract}
During dropwise condensation from the ambient environment, water vapor present in air must diffuse to the surface of each droplet. The spatial distribution of water vapor in the local surroundings of each individual droplet determines the total condensation rate. However, available models for dropwise condensation in humid air assume that such systems of droplets grow either as an equivalent film or that the growth of each droplet is completely isolated; the interactions between droplets are poorly described and, consequently, predictions of total condensation rates may mismatch experimental observations. This paper presents a reduced-order analytical method to calculate the condensation rate of each individual droplet within a group of droplets on a surface by resolving the vapor concentration field in the surrounding air. A point sink superposition method is used to account for the interaction between droplets without requiring solution of the diffusion equation for a full three-dimensional domain containing all of the droplets. For a simplified scenario containing two neighboring condensing droplets, the rates of growth are studied as a function of the inter-droplet distance and the relative droplet size. For representative systems of condensing droplets on a surface, the total condensation rates predicted by the reducedorder model match numerical simulations to within $15 \%$. The results show that assuming droplets grow as an equivalent film or in a completely isolated manner can severely overpredict condensation rates.
\end{abstract}




\section{Nomenclature}

c vapor concentration

D diffusion coefficient

$j \quad$ mass flux

$N \quad$ number of droplets in the system

$p \quad$ center-to-center pitch between the droplets

$\vec{r} \quad$ position vector

$R \quad$ single droplet radius

$\langle R\rangle \quad$ average droplet radius

$R H \quad$ relative humidity

$t \quad$ time

$T \quad$ temperature

$(x, y, z) \quad$ Cartesian coordinates

\section{Greek}

$(\alpha, \beta, \phi) \quad$ toroidal coordinates

$\xi \quad$ integration variable

$\theta \quad$ contact angle

$\mu \quad$ power law exponent

$\rho \quad$ density

$\sigma \quad$ sink density intensity per unit area

$\tau \quad$ integration variable

$v \quad$ normalized vapor concentration field 


\section{Subscripts}

c contact area between the drop and the substrate

iso isolated from neighboring droplets

$l \quad$ liquid

$s \quad$ at the surface of the drop

sys in the presence of neighboring droplets (system)

$\infty \quad$ far field

\subsection{Introduction}

Collection of water by condensation from humid air is a commonly occurring natural phenomenon also present in several engineering systems. In nature, diverse plants and animals have adapted to survive in arid regions by harvesting water from moist air [1,2]. Condensation from atmospheric air has several practical applications and is an attractive alternative supply of fresh water in arid regions [3,4]. However, due to the limited amount of water vapor present in the air, requirement of cooling power [5], and the sensitivity of condensation to environmental conditions [3,6], accurate models that predict water harvesting capacity are critical to the design of harvesting systems for maximum yield [3,7,8]. Although, enhancing mass transfer during condensation in humid air is a topic that has been previously studied $[9,10]$, modeling approaches still require further development to account for various factors in the prediction of water harvesting performance, such as ambient relative humidity [11], sub-cooling temperature, surface properties (i.e., surface roughness), and contact angle [12]. Improved modeling approaches would allow for more accurate design and scaling of water harvesting systems.

During dropwise condensation at a set of fixed conditions (i.e., ambient temperature, ambient relative humidity, and surface sub-cooling temperature), the characteristics of droplet growth are time- and space-dependent. These variations are attributed to differences in the vapor concentration field surrounding each droplet, which vary cyclically from initial nucleation of a droplet to eventual roll-off. The spatiotemporal droplet growth has been often characterized by three stages [13-15]. In the first stage $(i)$, drops are distributed homogeneously and the distance 
between the drops is relatively larger than the average radius of the droplets. A common simplification is to assume that the droplets grow as if they are isolated from one another. As time progresses and droplets grow larger, the distance between droplets decreases. When the length of the vapor concentration profiles scales to the distance between drops, and the vapor distribution profiles overlap, it has been approximated that this closely-packed set of similarly-sized droplets can be approximated as a liquid film. In the second stage (ii), droplets coalesce, leading to a constant surface area coverage and self-similar growth pattern. Even though the size of the droplets can be different over a broad range, it is still commonly assumed that droplet growth can be described with a filmwise-like growth approximation. As the droplets grow and the number of coalescence events decreases, the distance between droplets will increase, causing new droplets to nucleate in the bare space between the larger droplets. Two families of drops will be present on the surface, small droplets corresponding these re-nucleated droplets and large droplets that originated at earlier stages. Later, in the final stage (iii), droplets approach the capillary length and will roll off the surface due to gravity. In summary, within each cycle, droplets increase in size by two primary mechanisms: direct condensation of vapor at the liquid-gas interface and coalescence of multiple droplets. The growth of a system of droplets by condensation has been often described by the limiting theoretical cases of filmwise-like growth for closely-spaced droplets that compete for vapor in their surroundings or isolated growth of droplets spaced far apart.

While these simplified descriptions of the condensation process are well accepted, and have historically been an invaluable tool for explaining empirical observations, there are some specific growth characteristics that cannot be reasonably captured. For example, it has been observed that equally sized neighboring droplets growing in close proximity to each other have condensation rates up to $40 \%$ lower than predicted by isolated droplet growth models [12] and small droplets experience a reduced rate of growth when in close proximity to large droplets [16]; both phenomena are attributed to blocking of the lateral flux of vapor to the droplets. Local vapor distribution has also been shown to play a fundamental role in understanding several freezing phenomena such as inter-droplet ice bridging [17,18] and frost halos [19]. Also, geometric discontinuities such as surface edges and corners can also lead to changes in the vapor concentration filed around the droplets which can dramatically affect the rate of growth. Medici et al. [20] observed that droplets near the edges or a corner of a substrate grew $\sim 500 \%$ faster than 
a droplet near the center. In general, the total rate of condensation on a surface depends on the distribution of vapor in the surroundings that is governed by interactions within the entire set of droplets on the surface as well as the substrate boundaries, which is time- and space-dependent; modeling approaches are required that can capture all of these transport complexities.

At a given instant during the condensation process, the condensation rate of each individual droplet on a surface could be calculated by numerically solving the diffusion equation for the entire domain; however, due to the large number of differing size droplets, numerically modeling dropwise condensation in this multi-scale domain is rarely employed. Analytical solutions for the diffusion equation are available for the case of a single isolated droplet [21], but this approach is only applicable for the condensation of droplets separated by large distances [12], which might rarely occur under practical conditions. To account for interactions between droplets in the vapor concentration field, superposition methods have been used to describe the evaporation of suspended droplets during combustion [22]. Annamalai et al. [23] assumed suspended drops can be treated as point vapor sources to develop a point source method that solves for the evaporation rate of multiple droplets by superimposing Maxwell's solutions for the evaporation of individual droplets. The literature lacks a model that takes into account droplet interactions when solving the vapor concentration field during dropwise condensation of humid air on a substrate.

This work develops a model to calculate the condensation rate during dropwise condensation from humid air by incorporating the interactions between all droplets using a point sink method to superpose solutions of the vapor-diffusion-driven condensation of each individual droplet. The model requires as input the thermodynamic conditions (substrate temperature, air temperature, and relative humidity of the air), the location of the droplets, and the droplet contact angle. The model is used to predict condensation of a pair of neighboring droplets for a range of inter-droplet distances and relative sizes; the results are compared to the predictions of a three-dimensional numerical solution of the diffusion equation. This comparison is also drawn for the prediction of the condensation rate of each individual droplet in representative systems of many droplets extracted from previously reported experimental images. The model captures blocking effects due to differences in droplet size, as well as the effects of the complex spatial distribution of vapor concentration, on the condensation rate of each droplet. This compact model achieves an intermediate complexity that retains good accuracy compared to the complete numerical solution 
of vapor concentration field, while accounting for critical additional physical phenomena compared to alternative analytical modeling approaches.

\subsection{Model Description}

\subsection{Condensation of an isolated droplet}

For a droplet that is resting on a cooled surface kept at a constant temperature $\left(T_{s}\right)$ below the temperature $\left(T_{\infty}\right)$ of the surrounding air at some relative humidity $(R H)$, condensation of water vapor will occur on droplet surface. In quiescent air, growth of the droplet is governed by the diffusion of water molecules to this liquid-vapor interface. The concentration of water vapor $c(\vec{r}, t)$ in the air surrounding the droplet obeys the diffusion equation:

$$
\frac{\partial c(\vec{r}, t)}{\partial t}=D \nabla^{2} c(\vec{r}, t)
$$

The diffusion time scale for small condensing drops is of the order of $R^{2} / D \approx 10^{-7} \mathrm{~s}[21](e . g$, for an initial drop radius of $\sim 5 \mu \mathrm{m}$ and a diffusion coefficient of $25.4 \times 10^{-6} \mathrm{~m}^{2} / \mathrm{s}$ for water vapor in air), which is significantly smaller than the time scale of the growth of a droplet during condensation. Thus, the vapor concentration field adjusts rapidly compared to changes in the drop shape and the droplet condensation process can be assumed to be quasi-steady. Equation (1) can be rewritten as:

$$
\nabla^{2} c=0
$$

The solution to this Laplace equation must satisfy the boundary conditions at the substrate, at the surface of the droplet, and far away from the droplet. The vapor concentration at the surface of the droplet $c\left(\vec{r}_{s}\right)=c_{s}$ is assumed to be equal to the saturated vapor pressure at the droplet surface temperature. This assumption is valid when there is a small temperature drop across the height of the droplet during diffusion-driven condensation; the additional effects of external convection [24], thermocapillary flows [25], and the release of latent heat [26] on the interface temperature are not 
considered. In the far field, the vapor concentration $c(\vec{r} \rightarrow \infty)=c_{\infty}$ corresponds to the vapor pressure at the air temperature and relative humidity. There is zero mass flux normal to the substrate (i.e. $\partial c /\left.\partial z\right|_{z=0}=0$ ). For an analogous process of droplet evaporation, where the boundary conditions are the same but the mass flux direction changes, Popov [21] provided a closed-form solution for the concentration field in toroidal coordinates. Ucar and Erbil [12] and GuadarramaCetina et al. [27] later used this solution to describe droplets growing by condensation on polymeric surfaces under the assumption that they were isolated from one another. Rewriting the solution for an evaporating droplet, the distribution of vapor in the area surrounding a condensing droplet is given by:

$$
\frac{c_{\infty}-c(\alpha, \beta)}{c_{\infty}-c_{s}}=\sqrt{2(\cosh (\alpha)-\cos (\beta))}\left\{\int_{0}^{\infty} \frac{[\cosh (\theta \tau)][\cosh ((2 \pi-\beta) \tau)]}{[\cosh (\pi \tau)][\cosh ((\pi-\theta) \tau)]} P_{-1 / 2+i \tau}(\cosh (\alpha)) d \tau\right\}
$$

where $\alpha$ and $\beta$ are toroidal coordinates $(0 \leq \alpha \leq \infty$ and $\pi-\theta<\beta<\pi+\theta), \theta$ is the contact angle of the droplet with the substrate, and $P_{-1 / 2+i \tau} \cosh (\alpha)$ is the Legendre function of the first kind given by:

$$
P_{-1 / 2+i \tau}(\cosh (\alpha))=\frac{2}{\pi} \operatorname{coth}(\pi \tau) \int_{\alpha}^{\infty} \frac{\sin (\tau \xi)}{\sqrt{2 \cosh (\xi)-2 \cosh (\alpha)}} d \xi
$$

Equation (3) requires two numerical integrations with respect to $\tau$ and $\xi$. For the special case of a contact angle of the droplet set $\theta=90 \mathrm{deg}$ in equation (3), the solution of equation (3) in toroidal coordinates converges to the solution of equation (2) in Cartesian coordinates:

$$
\frac{c_{\infty}-c(\alpha, \beta)}{c_{\infty}-c_{s}}=\sqrt{\frac{\cosh \alpha-\cos \beta}{\cosh \alpha+\cos \beta}}=\frac{R_{c}}{|\vec{r}|}
$$


where $|\vec{r}|=\sqrt{x^{2}+y^{2}+z^{2}}$ is the Euclidian distance from the origin to any point in the domain. In general, for any contact angle, the rate of growth of a single drop $\dot{m}_{i s o}$ can be obtained by integrating the flux of vapor from the surroundings at the surface of the droplet $j(r)$ :

$$
\dot{m}_{i s o}=\frac{d m}{d t}=\rho_{l} \frac{d V}{d t}=\rho_{l} \int j(r) d s=\pi R_{c} D\left(c_{\infty}-c_{s}\right) f(\theta)
$$

where $R_{c}$ is the contact radius of the droplet. The condensation rate from the surface of the drop $j(r)$ and $f(\theta)$ are given by:

$$
\begin{gathered}
j(r)=\frac{D\left(c_{\infty}-c_{s}\right)}{R_{c}}\left[\frac{1}{2} \sin \theta+\sqrt{2}(\cosh \alpha+\cos \theta)^{3 / 2} \int_{0}^{\infty} \frac{\cosh \theta \tau}{\cosh \pi \tau} \tanh [(\pi-\theta) \tau] d \tau\right] \\
f(\theta)=\frac{\sin \theta}{1+\cos \theta}+4 \int_{0}^{\infty} \frac{1+\cosh 2 \theta \tau}{\sinh 2 \pi \tau} \tanh [(\pi-\theta) \tau] d \tau
\end{gathered}
$$

where for small contact angles $f(\theta \rightarrow 0) \rightarrow 1$, while for large contact angles $f\left(\theta \rightarrow 180^{\circ}\right) \rightarrow \infty$.

\subsection{Point sink superposition method for vapor-diffusion-driven dropwise condensation}

Due to the linearity of the Laplace equation, the solution for the vapor concentration field surrounding a system of droplets can be described as a linear combination of the solutions for individual drops. A point sink superposition method treats each condensing droplet as a point vapor sink located at the center of the droplet. The model requires as inputs the sink intensities as if they were single isolated droplets in conjunction with the size and spatial distribution of the droplets; the substrate temperature and the contact angle of the droplet are also required. Some of the additional assumptions are inherited from the single-droplet condensation model introduced in Section 2.1: (i) there is negligible thermal resistance across the droplet (i.e., the temperature at the droplet surface is equal to the temperature of the substrate), (ii) vapor transport to the surface is governed by diffusion, and (iii) the condensation process can be treated as quasi-steady. In the 
two subsequent sections, the point sink method for a single condensing drop and systems of condensing droplets are introduced.

\subsubsection{Point sink method for a single condensing droplet on a substrate}

For a single point sink, the governing equation (2) transforms to a Poisson equation given by:

$$
\nabla^{2} c(\vec{r})=\sigma \delta\left(\vec{r}-\vec{r}_{j}\right)
$$

where $\delta\left(\vec{r}-\vec{r}_{j}\right)$ is the Dirac delta function representing a sink with density per unit area $\sigma$ located at a point $\vec{r}=\vec{r}_{j}$. The distribution of vapor in the surroundings of a point sink can be calculated by integrating equation (9) from $\vec{r}=0$ to and arbitrary location $\vec{r}$ :

$$
c_{\infty}-c(\vec{r})=\frac{\sigma}{|\vec{r}|} .
$$

If the point sink is assumed to capture vapor as a droplet located at the center of a hemispherical cap, the mass absorbed by the sink should be equal to the mass crossing the area of the hemispherical cap with contact angle $\theta$; for the case of a droplet with contact angle $\theta=90 \mathrm{deg}$, the intensity is equal to $\sigma=\dot{m}_{i s o} / 2 \pi D=R_{c}\left(c_{s}-c_{\infty}\right)$ as predicted from equation (5). In general, for any contact angle the sink intensity per unit area can be derived from equation (6) and it is given by $\sigma=\dot{m}_{i s o} / \pi f(\theta) D$. Equation (10) provides the vapor concentration field in the surroundings of a single point sink with the intensity of a vapor-diffusion-driven condensing droplet with contact angle $\theta$.

\subsubsection{Point sink superposition method for multiple condensing droplets on a substrate}

For a system with $N$ sinks at arbitrary locations $\vec{r}_{j}$ with $j=1,2,3 \ldots, N$ having the condensation intensity of droplet of with contact angle $\theta$, the concentration of the vapor at a location $\vec{r}$ in the domain due to the $N$ humidity sinks is obtained from the superposition of the concentration contours produced by each humidity sink (developed in Section 2.2.1) and is given by: 


$$
c_{\infty}-c(\vec{r})=\sum_{j=1}^{N} \frac{\dot{m}_{s y s, j}}{\pi D f(\theta)} \frac{1}{\left|\vec{r}-\vec{r}_{j}\right|},
$$

where $\left|\vec{r}-\vec{r}_{j}\right|$ is the distance from an arbitrary location $\vec{r}$ to the location of the $j^{\text {th }}$ humidity sink located at $\vec{r}_{j}$, and $\dot{m}_{s y s, j}$ is the rate of condensation of the $j^{\text {th }}$ humidity sink in the system.

It is of interest to calculate the effect that an array of surrounding sinks would have on the condensation of a single droplet in the system. Let a point sink be replaced by a droplet $i$ at the location $\vec{r}_{i}$, while all the other $j$ locations are occupied by surrounding point sinks. The location of this droplet's surface is described by a vector $\vec{r}_{s i}$ from the droplet center and the concentration of vapor at the surface of the droplet can be obtained from substituting $\vec{r}=\vec{r}_{i}+\vec{r}_{s i}$ in equation (11) which results in:

$$
c_{\infty}-c_{s}\left(\vec{r}_{i}+\vec{r}_{s i}\right)=\sum_{j=1}^{N}\left(\frac{\dot{m}_{s y s, j}}{\pi D f(\theta)} \frac{1}{\left|\vec{r}_{i}+\vec{r}_{s i}-\vec{r}_{j}\right|}\right)
$$

Because the temperatures of all droplets are assumed to be equal (at the substrate temperature), the vapor concentration at the surface of the droplet is the same for every droplet in the system, $c_{s}\left(\vec{r}_{1}+\vec{r}_{s 1}\right)=c_{s}\left(\vec{r}_{2}+\vec{r}_{s 2}\right)=. .=c_{s}\left(\vec{r}_{i}+\vec{r}_{s i}\right)$. While the magnitude of $\vec{r}_{s i}$ is different for each point on the droplet surface, it can be assumed that this magnitude is small in comparison with the distance between the droplets $\left|\vec{r}_{s i}\right| \square\left|\vec{r}_{i}-\vec{r}_{j}\right|$. If we further assume $\left|\vec{r}_{s i}\right| \approx R_{c i}$ for the term $i$ in the summation, then equation (12) can be rewritten as a system of $N$ equations:

$$
c_{\infty}-c_{s}=\frac{\dot{m}_{s y s, i}}{\pi D f(\theta) R_{c i}}+\sum_{j=1, j \neq i}^{N}\left(\frac{\dot{m}_{s y s, j}}{\pi D f(\theta) R_{c i}} \frac{R_{c i}}{\left|\vec{r}_{i}-\vec{r}_{j}\right|}\right),
$$


where $i=1,2, \ldots N$ represents each droplet location on the substrate, and $j=1,2, \ldots N$ represents the surrounding humidity sinks at each droplet location, as shown in Figure 1.

The solution of the condensation rate of each droplet in the system is simplified if dropletto-droplet interaction is cast as a correction factor $\eta$ given by the ratio between the condensation rate of the droplet within the system of multiple droplets versus the condensation rate of the droplet as if it was isolated:

$$
\eta=\frac{\dot{m}_{s y s}}{\dot{m}_{i s o}}
$$

where $\eta=1$ indicates that a droplet will grow as if it were isolated. Because all the droplets are assumed to have the same vapor concentration at their surfaces, by dividing equation (13) by $\left(c_{\infty}-c_{s}\right)$ and using the definition of the correction factor given in equation (14) the system of equations (13) can be rewritten in terms of the correction factor $\eta$ as:

$$
\eta_{i}+\sum_{j=1, j \neq i}^{N} \eta_{j}\left(\frac{R_{c j}}{\left|\vec{r}_{i}-\vec{r}_{j}\right|}\right)=1
$$

The solution of the system of equations provides the correction factor for each droplet; from equation (14) the condensation rate for each droplet can be obtained. Introducing the normalized concentration field as:

$$
v(\vec{r})=\frac{c_{\infty}-c(\vec{r})}{c_{\infty}-c_{s}},
$$

and dividing equation (11) by $c_{\infty}-c_{s}$, the potential concentration field can be rewritten in terms of the correction factor as: 


$$
v(\vec{r})=\sum_{j=1}^{N}\left(\frac{\dot{m}_{s y s, j}}{\pi D f(\theta)\left(c_{\infty}-c_{s}\right)\left|\vec{r}-\vec{r}_{j}\right|}\right)=\sum_{j=1}^{N}\left(\frac{\eta_{j} R_{c j}}{\left|\vec{r}-\vec{r}_{j}\right|}\right)
$$

Thus, the normalized concentration contour field is obtained once the correction factors have been determined from equation (15).

Equations (14) to (17) provide a closed-form solution for the instantaneous condensation rate and normalized local vapor concentration for vapor-diffusion-driven dropwise condensation from humid air by the point sink superposition method.

\subsection{Results}

\subsection{Condensation of a pair of droplets}

This section compares the condensation rates predicted for a pair of neighboring droplets obtained using the point sink superposition method developed in Section 2.0 against a threedimensional numerical solution of the diffusion equation. The case considers two droplets resting on a substrate with a contact angle of $\theta=120 \mathrm{deg}$, separated by a pitch $p$ and contact radii $R_{c 1}$ and $R_{c 2}$. The condensation rates are predicted for a range of contact radii varying from $10 \mu \mathrm{m}$ to $310 \mu \mathrm{m}$ and inter-droplet distances from $120 \mu \mathrm{m}$ to $1140 \mu \mathrm{m}$. The temperature of the droplets is assumed to be equal to the substrate temperature $T_{\text {sub }}=5{ }^{\circ} \mathrm{C}$, and thus the vapor concentration at the surface of the droplet is $c_{s}=0.0035 \mathrm{~kg} / \mathrm{m}^{3}$. The concentration at the outer surface of the domain is taken at $c_{\infty}=0.00124 \mathrm{~kg} / \mathrm{m}^{3}$ for $70 \%$ relative humidity at an air temperature of $T_{\text {air }}=20{ }^{\circ} \mathrm{C}$.

The condensation rate correction factors from the point sink method can be obtained by solving the system of equations in the Equation (15) for two droplets as:

$$
\eta_{1}=\frac{\left(1-\frac{R_{c 2}}{p}\right)}{\left(1-\frac{\left(R_{c 1}\right)\left(R_{c 2}\right)}{p^{2}}\right)} \quad \eta_{2}=\frac{\left(1-\frac{R_{c 1}}{p}\right)}{\left(1-\frac{\left(R_{c 1}\right)\left(R_{c 2}\right)}{p^{2}}\right)}
$$

By substituting the corrections factors given into Equation (17), the normalized concentration field for two drops can be calculated as: 


$$
v(\vec{r})=\frac{\eta_{1} R_{c 1}}{\left|\vec{r}-\vec{r}_{1}\right|}+\frac{\eta_{2} R_{c 2}}{\left|\vec{r}-\vec{r}_{2}\right|}
$$

The numerical simulations are performed by using the finite volume schemes implemented in ANSYS Fluent 17.2 [28]. Figure 2 (a) shows the meshed spherical cap used as computational domain; the pair of droplets, which are much smaller than the overall domain, are located on the substrate in the center as shown in the zoomed view in Figure 2 (b). As boundary conditions, the vapor concentration was prescribed at the outer surface of the domain and on the surface of each drop, and a zero flux condition was prescribed on the substrate surface. The shape and size of the domain were chosen to ensure domain-independent results. Considering all of the different cases, a typical domain used a mesh with $\sim 10^{6}$ elements; a mesh independence analysis, with local refinements near the surfaces of the droplets in the regions of high concentration gradients, was performed to confirm that results of the numerical calculations were independent of the size of the elements used. The criteria for convergence was set at a normalized absolute error of $\sim 10^{-12}$.

The effects of inter-droplet distance on the condensation rate are first explored by changing droplet pitch between two droplets of the same size $R_{c}=R_{c 1}=R_{c 2}$. Figure 3 (a) shows the correction factor $\eta=\eta_{1}=\eta_{2}$ as function of the droplet pitch for all of the sizes considered. The predictions using the point sink method (shown as solid lines) closely matches the results of the numerical simulations (shown as symbols). The relative error between these two values, for all the cases considered, remains below $4 \%$. For a selected case, Figure 4 shows the normalized vapor concentration field $v(\vec{r})$ at the substrate plane in a region near the two droplets; local agreement between the model and the numerical simulations is observed in the field.

Each droplet will grow as if it were completely isolated for a correction factor equal to unity; in the limit of the droplet pitch being very large $(p \rightarrow \infty)$, the droplets will have no effect on each other $(\eta \rightarrow 1)$. All of the cases shown in Figure 3 tend to $\eta=1$ with increasing pitch; only for relatively extreme separation distances (e.g., droplets separated by a pitch one hundred times their radii) does this value become near unity. At a given pitch, the correction factor reduces as the size of the droplets increase. If the correction factor is replotted as a function of the non-dimensional ratio between the pitch of the droplet pair and their contact radii, as shown in Figure 3 (b), then all 
of the data from Figure 2 (a) fall onto a single master curve for the correction factor. From Figure 3 (b), it can again be observed that the correction factor increases with an increasing ratio between the droplet pitch and their contact radii. This curve can be used to define a threshold separation distance at which it can be assumed that droplets of the same size have negligible interaction with each other. For example, a value of $p / R_{c}>25$ (i.e., droplets separated at a distance more than 25 times greater than their contact radii) the correction factor is $\eta>0.95$.

The reduction of the condensation rate as the droplets become closer (i.e., as the pitch decreases) is further examined in Figure 5 by plotting contours of the normalized concentration field $v(\vec{r})$ around the two droplets given by Equation (19) at the plane that coincides with the substrate at $z=0$. The panels of Figure 5 show the results for two droplets with contact radii $R_{c}$ $=60 \mu \mathrm{m}$ at different pitches. A zone of depleted vapor emerges between the droplets that significantly reduces the concentration gradient normal to the droplet surface in the direction toward the other droplet (compared to the opposing direction). As the distance between the droplets decreases, this depletion zone becomes more severe as the maximum concentration of vapor decreases; hence, the condensation rate of each drop will be further reduced as they are brought closer. From the example case shown in Figure 5, two droplets with $R_{c}=60 \mu \mathrm{m}$ separated by a distance equal to $p=180 \mu \mathrm{m}$ would condense $25 \%$ less rapidly compared to the same size isolated droplet. Even for pitches that are 9 times larger than the droplet radius, the condensation rate would be reduced by $9.5 \%$.

The relative sizes of the droplets in the pair also plays an important role in affecting the condensation behavior. This is analyzed by considering a droplet with contact radius $R_{c 1}=10 \mu \mathrm{m}$ that is in the surroundings of a relatively larger droplet with a contact radius $R_{c 2}$. Figure 6 (a) and (b) respectively show the condensation rate correction factors for these two droplets, $\eta_{1}$ and $\eta_{2}$ for $R_{c 2}$ increasing from $10 \mu \mathrm{m}$ to $310 \mu \mathrm{m}$, as function of droplet pitch. The relative error between the point sink superposition method prediction (solid lines) and the numerical simulation results (data points) increases as the ratio between the droplet radii increases and the pitch is reduced; for all the cases the relative error was larger for the smaller drop.

There is a very significant reduction in the condensation rate of the small droplet as the size of the larger neighboring droplet increases (see Figure 6 (a)). For example, at $p=600 \mu \mathrm{m}$, for the 
larger droplet increasing in size from $10 \mu \mathrm{m}$ to $310 \mu \mathrm{m}$, the correction factor of the smaller droplet decreases from $\eta_{1}=0.98$ to $\eta_{1}=0.41$. Comparatively, the correction factor for the larger droplet is universally $\eta_{2}>0.98$ for all the cases at this pitch. This can be further explained by Figure 7, which shows the normalized vapor concentration field $v(\vec{r})$ at the substrate for $R_{c 1}=10 \mu \mathrm{m}$ and $R_{c 2}=60 \mu \mathrm{m}$ at different pitches. The concentration field is almost entirely governed by the larger droplet, which causes the smaller droplet to lie in a zone of depleted vapor. As the large droplet comes closer, the smaller droplet has a reduced concentration of vapor available in its immediate surroundings; however, the concentration field observed from the perspective of the larger droplet is relatively unaffected.

Reduced condensation rates for small droplets in the surroundings of relatively larger droplets has previously been observed in experiments; Leach et al. [16] reported small droplets near larger droplets grew 20\% slower compared to more isolated droplets of the same size. Depletion of vapor has also been reported as a factor causing the inhibition of droplet nucleation on the bare substrate nearby large droplets during condensation [27].

\subsection{Condensation in systems of many droplets}

This section first compares the overall condensation rates and water vapor distribution obtained for a system of multiple droplets using the point sink superposition method against a numerical simulation of the same system. The computational domain, boundary conditions, and implementation approach used for the numerical simulations are similar to those described in Section 3.1, but updated to accommodate more than two droplets at the center of the domain. Secondly, the point sink superposition method is used to predict the condensation rate of randomly distributed systems of droplets having size distributions resembling previously reported experimental data [11]; these predicted rates are compared against alternative reduced-order prediction methods.

To implement the point sink superposition method, the locations and sizes of the droplets in the system are inserted into equation (15), and the correction factor and condensation rate of each droplet are obtained by solving the system of equations. Subsequently, the normalized local vapor concentration field at the substrate is obtained by substituting the correction factors into equation (17). The thermodynamic conditions (i.e., substrate temperature, air temperature, relative humidity and vapor concentrations) are the same as defined previously in Section 3.1. 


\subsubsection{Comparison of point sink superposition method versus prediction via numerical simulations}

The point sink superposition method predictions are first compared with numerical simulations to verify the superposition approach for a system of multiple droplets. The system of droplets used for this comparison is shown in Figure 8, which contains 16 droplets and resembles a time during condensation when the drop size distribution is broad and there are larger bare spaces between the droplets. The vapor concentration field is shown for the numerical simulation in Figure 8 (a) and for the point sink method in Figure 8 (b); the vapor distributions around the droplets obtained from both models are observed to be similar. The ability of the point sink superposition method to match the numerical simulations can be further investigated by comparing the condensation rates of each individual droplet, as well as the total condensation rate, predicted by both of the models, as summarized in Table 1. The total condensation rate error was found to be $\sim 14 \%$, and the error for individual droplets was generally on the same order. These findings are consistent with the comparisons presented for the case of two condensing droplets shown in Section 3.1. Because the vapor concentration distribution around the droplets determines their condensation rate, droplets

of similar size have a higher condensation rate when they are nearer the edge of the system. For example, droplet 16 in the system of droplets shown in Figure 8, which is located near the edge of the system, has a larger condensation rate compared to droplet 8 located near the center. The area surrounding the droplets deep in the center of the array is almost entirely depleted of vapor, and all droplets interact and significantly influence one another. This behavior, which causes droplets near the edge of the system to grow faster than droplets near the center, has previously observed by Medici et. al. [20]. Another unique validation case for a different system of droplets is provided in the Supplementary Materials.

\subsubsection{Comparisons of point sink superposition method versus prediction via conventional reduced-order models}

The condensation rates predicted for a system of droplets using the point sink superposition method, which accounts for the complete vapor diffusion behavior, is compared against condensation rates estimated using the highly simplified approaches of assuming completely isolated droplet growth or filmwise-like growth behavior, as is often employed in the literature $[16,20]$. The condensation rate of isolated droplet growth is obtained from equation (6), while the condensation rate of filmwise-like growth is obtained from a simplified one-dimensional diffusion 
resistance analysis. This filmwise growth model assumes that a system of closely-spaced droplets behaves as a film of equivalent condensate volume. By solving equation (2) in the direction normal to the surface of the film and from scaling the extension of the concentration profile to a region where the diffusion of vapor is dominant, the condensation rate of the film can be obtained as previously reported by Medici et. al [20].

Two different characteristic droplet systems are considered for this analysis, as shown in Figure 9. The system shown in Figure 9 (a) resembles the earliest stages of growth (i.e., regime $i$ as discussed in Section 1.0) after nucleation when the droplets have similar sizes and are closely spaced, while Figure 9 (b) resembles a later stage during condensation when there are a significant number of coalescence events (i.e., regime $i i$ as discussed in Section 1.0) that leads to a broader droplet size distribution. These systems of droplets are randomly generated to achieve the same droplet distribution characteristics as observed in our previous experiments [11]. The point sink superposition model is evaluated considering the entire domain area in Figure 9. To avoid the influence of edge effects, the comparisons only consider the condensation behavior within a region near the center of the system (viz. within the dashed square shown in Figure 9); it was separately confirmed that this region is not affected by the edges of the domain, and therefore representative of the condensation behavior on an infinite plane.

The total condensation rates of the droplets enclosed by the dashed squares shown in Figure 9 are summarized in Table 2 (a). The total condensation rate estimated by the isolated droplet growth model severely overpredicts condensation rates obtained by the point sink superposition method for both systems. During evaluation of the point sink superposition model, the average condensation rate correction factor for the droplets within the area enclosed by the dashed square shown in Figure 9 (a) was found to be $\left\langle\eta_{i}\right\rangle=0.13$. Droplets in closer proximity to neighboring droplets grow at smaller rates compared to droplets that are spaced further away from their neighbors. For example, the condensation correction factor of the droplet $a 1$ in Figure 9 (a) is lesser than the similarly sized droplet $a 2$ (see Table 2 (b)). Large bare spaces between the droplets promote higher condensation rates for individual droplets because vapor can diffuse vertically toward the substrate and then laterally toward the peripheral of the droplets; conversely, droplets in the neighborhood block the lateral diffusion of the vapor, causing depletion of vapor, as discussed in Section 3.1. In comparison to the system of droplets shown in Figure 9 (a), the system of droplets shown in Figure 9 (b) has a smaller average condensation correction factor of $\left\langle\eta_{i i}\right\rangle=$ 
0.07 due to a reduction of the distance between droplets. There is also a broader distribution of sizes and vapor in the surrounding of relatively small droplets is depleted by large droplet neighbors. For instance, the small droplet $b 1$ shown in Figure 9 (b) has a condensation correction factor equal to $\eta=0.02$, compared to $\eta=0.10$ for a larger droplet $b 2$. While filmwise-like growth is commonly used to characterize droplet growth in such systems, the filmwise-like condensation model can lead to errors in prediction because its intrinsic assumption of an equivalent condensate volume yields a condensing interface with a different area compared to the total surface area of the droplets; in addition, the filmwise-like condensation model heavily relies on scaling approximations to predict the concentration profile. Even though the filmwise-like condensation model provides a better prediction compared to assuming that droplets grow as if they were isolated, the filmwise-like growth model overestimates the total condensation rate by $\sim 60 \%$ compared to the prediction of the point sink superposition method for the specific systems shown in Figure 9.

\subsection{Conclusions}

This paper presents a methodology to calculate the condensation rates of each individual droplet within large systems of many droplets during vapor-diffusion-driven dropwise condensation from humid air. This methodology treats each individual droplet as a point humidity sink so as to allow superposition of the solutions to the diffusion equation for each individual drop. The model thereby captures the interaction between all droplets within the system, accounting for spacing between droplets and their relative differences in size (such as the effect of large droplets on blocking water vapor flux toward small nearby droplets). This methodology is shown to accurately predict the total condensation rate and local vapor distribution for systems of droplets by validation against a three-dimensional numerical solution of the diffusion equation. In comparison with highly simplified droplet growth models, viz., assuming completely isolated droplets or filmwise-like growth, the critical importance of including droplet interaction effects is demonstrated by comparing to the total condensation rate calculated using the point sink method for droplet systems representative of dropwise condensation. 


\section{Acknowledgments}

The first author acknowledges the financial support provided by the Colombian Department for Science, Technology and Innovation (Colciencias) and the Colombia-Purdue Institute (CPI). Special thanks to Prof. Suresh V. Garimella in the School of Mechanical Engineering at Purdue University for technical discussions related to this work.

\section{References}

[1] C. R. Tracy, Laurence Nathalie, and K. A. Christian, "Condensation onto the Skin as a Means for Water Gain by Tree Frogs in Tropical Australia.," Am. Nat., vol. 178, no. 4, pp. 553-558, Oct. 2011.

[2] A. J. Hill, T. E. Dawson, O. Shelef, and S. Rachmilevitch, "The role of dew in Negev Desert plants," Oecologia, vol. 178, no. 2, pp. 317-327, Jun. 2015.

[3] M. Tomaszkiewicz, M. Abou Najm, D. Beysens, I. Alameddine, and M. El-Fadel, "Dew as a sustainable non-conventional water resource: a critical review," Environ. Rev., vol. 23, no. 4, pp. 425-442, Sep. 2015.

[4] H. Vuollekoski et al., "Estimates of global dew collection potential on artificial surfaces," Hydrol Earth Syst Sci, vol. 19, no. 1, pp. 601-613, Jan. 2015.

[5] D. Beysens et al., "Application of passive radiative cooling for dew condensation," Energy, vol. 31, no. 13, pp. 2303-2315, Oct. 2006.

[6] B. Gido, E. Friedler, and D. M. Broday, "Assessment of atmospheric moisture harvesting by direct cooling," Atmospheric Res., vol. 182, pp. 156-162, Dec. 2016.

[7] D. Beysens, I. Milimouk, V. Nikolayev, M. Muselli, and J. Marcillat, "Using radiative cooling to condense atmospheric vapor: a study to improve water yield," J. Hydrol., vol. 276, no. 1-4, pp. 1-11, May 2003.

[8] D. Milani, A. Abbas, A. Vassallo, M. Chiesa, and D. A. Bakri, "Evaluation of using thermoelectric coolers in a dehumidification system to generate freshwater from ambient air," Chem. Eng. Sci., vol. 66, no. 12, pp. 2491-2501, Jun. 2011.

[9] A. Lee, M.-W. Moon, H. Lim, W.-D. Kim, and H.-Y. Kim, "Water harvest via dewing," Langmuir, vol. 28, no. 27, pp. 10183-10191, Jul. 2012.

[10] P. S. Mahapatra, A. Ghosh, R. Ganguly, and C. M. Megaridis, "Key design and operating parameters for enhancing dropwise condensation through wettability patterning," Int. J. Heat Mass Transf., vol. 92, pp. 877-883, Jan. 2016.

[11] J. E. Castillo, J. A. Weibel, and S. V. Garimella, "The effect of relative humidity on dropwise condensation dynamics," Int. J. Heat Mass Transf., vol. 80, pp. 759-766, Jan. 2015.

[12] I. O. Ucar and H. Y. Erbil, "Use of diffusion controlled drop evaporation equations for dropwise condensation during dew formation and effect of neighboring droplets," Colloids Surf. Physicochem. Eng. Asp., vol. 411, pp. 60-68, Oct. 2012.

[13] J. L. Viovy, "Scaling description for the growth of condensation patterns on surfaces," Phys. Rev. A, vol. 37, no. 12, pp. 4965-4970, 1988. 
[14] D. Fritter, C. M. Knobler, and D. A. Beysens, "Experiments and simulation of the growth of droplets on a surface (breath figures)," Phys. Rev. A, vol. 43, no. 6, pp. 2858-2869, Mar. 1991.

[15] J. Blaschke, T. Lapp, B. Hof, and J. Vollmer, "Breath Figures: Nucleation, Growth, Coalescence, and the Size Distribution of Droplets," Phys. Rev. Lett., vol. 109, no. 6, Aug. 2012.

[16] R. N. Leach, F. Stevens, S. C. Langford, and J. T. Dickinson, "Dropwise Condensation: Experiments and Simulations of Nucleation and Growth of Water Drops in a Cooling System," Langmuir, vol. 22, no. 21, pp. 8864-8872, Oct. 2006.

[17] X. Chen et al., "Activating the Microscale Edge Effect in a Hierarchical Surface for Frosting Suppression and Defrosting Promotion," Sci. Rep., vol. 3, p. srep02515, Aug. 2013.

[18] J. B. Boreyko, R. R. Hansen, K. R. Murphy, S. Nath, S. T. Retterer, and C. P. Collier, "Controlling condensation and frost growth with chemical micropatterns," Sci. Rep., vol. 6, Jan. 2016.

[19] S. Nath and J. B. Boreyko, "On Localized Vapor Pressure Gradients Governing Condensation and Frost Phenomena," Langmuir, vol. 32, no. 33, pp. 8350-8365, Aug. 2016.

[20] M.-G. Medici, A. Mongruel, L. Royon, and D. Beysens, "Edge effects on water droplet condensation," Phys. Rev. E, vol. 90, no. 6, p. 062403, Dec. 2014.

[21] Y. O. Popov, "Evaporative deposition patterns: Spatial dimensions of the deposit," Phys. Rev. E, vol. 71, no. 3, p. 036313, Mar. 2005.

[22] W. A. Sirignano, "Advances in droplet array combustion theory and modeling," Prog. Energy Combust. Sci., vol. 42, pp. 54-86, Jun. 2014.

[23] K. Annamalai, W. Ryan, and S. Chandra, "Evaporation of Multicomponent Drop Arrays," J. Heat Transf., vol. 115, no. 3, pp. 707-716, Aug. 1993.

[24] S. Chavan et al., "Heat Transfer through a Condensate Droplet on Hydrophobic and Nanostructured Superhydrophobic Surfaces," Langmuir, vol. 32, no. 31, pp. 7774-7787, Aug. 2016.

[25] A. Phadnis and K. Rykaczewski, "The effect of Marangoni convection on heat transfer during dropwise condensation on hydrophobic and omniphobic surfaces," Int. J. Heat Mass Transf., vol. 115, no. Part B, pp. 148-158, Dec. 2017.

[26] Z. Pan, J. A. Weibel, and S. V. Garimella, "Influence of Surface Wettability on Transport Mechanisms Governing Water Droplet Evaporation," Langmuir, vol. 30, no. 32, pp. 97269730, Aug. 2014.

[27] J. Guadarrama-Cetina, R. D. Narhe, D. A. Beysens, and W. González-Viñas, "Droplet pattern and condensation gradient around a humidity sink," Phys. Rev. E, vol. 89, no. 1, p. 012402 , Jan. 2014.

[28] ANSYS, Inc., “ANSYS FLUENT 17.2 User’s Guide.” 2016. 


\section{Figures}

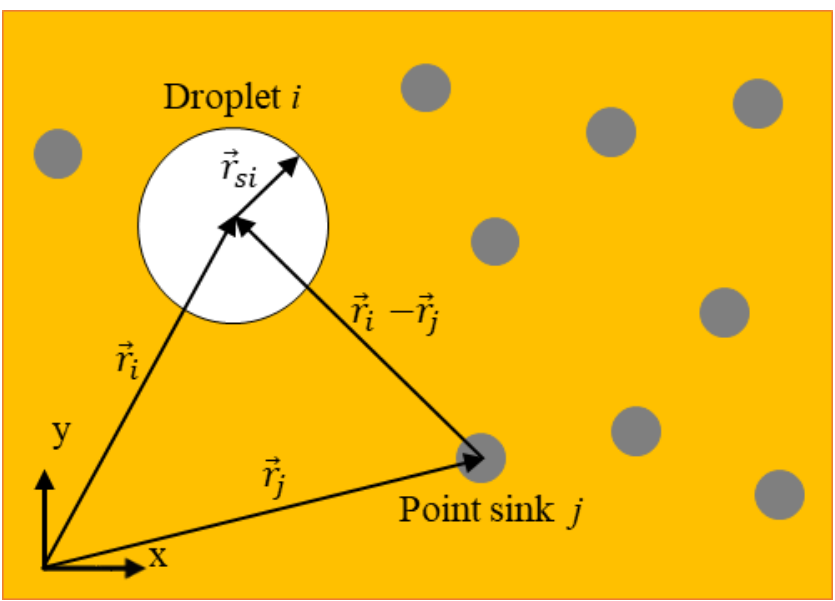

(a)

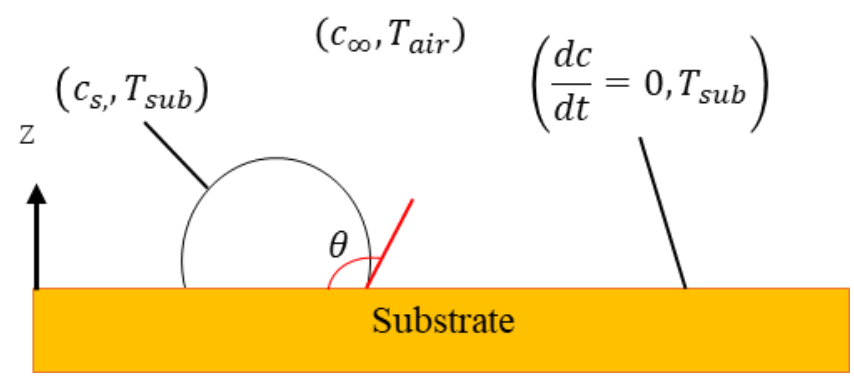

(b)

Figure 1. Schematic diagram of a droplet condensing on a substrate surrounded by multiple point humidity sinks: (a) the top view shows the vector position of the center droplet $i$ and surrounding humidity sinks at the locations of the other droplets $j$, and the (b) side view shows the substrate, droplets, and boundary conditions. 


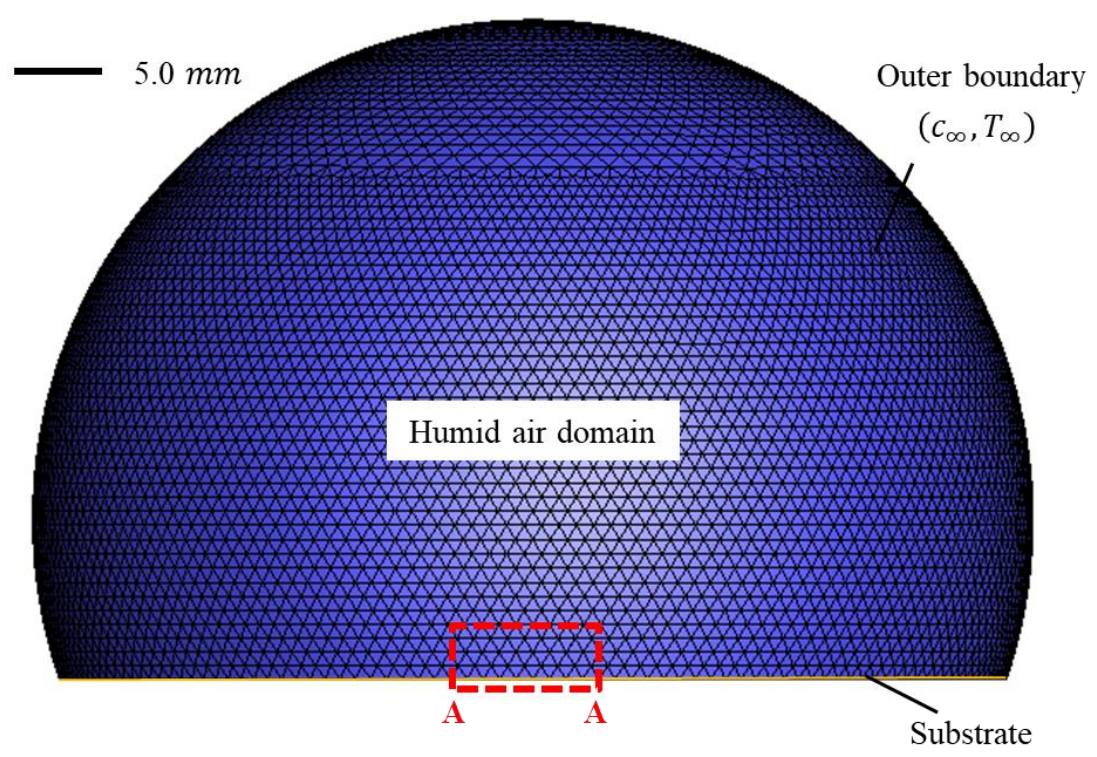

(a)

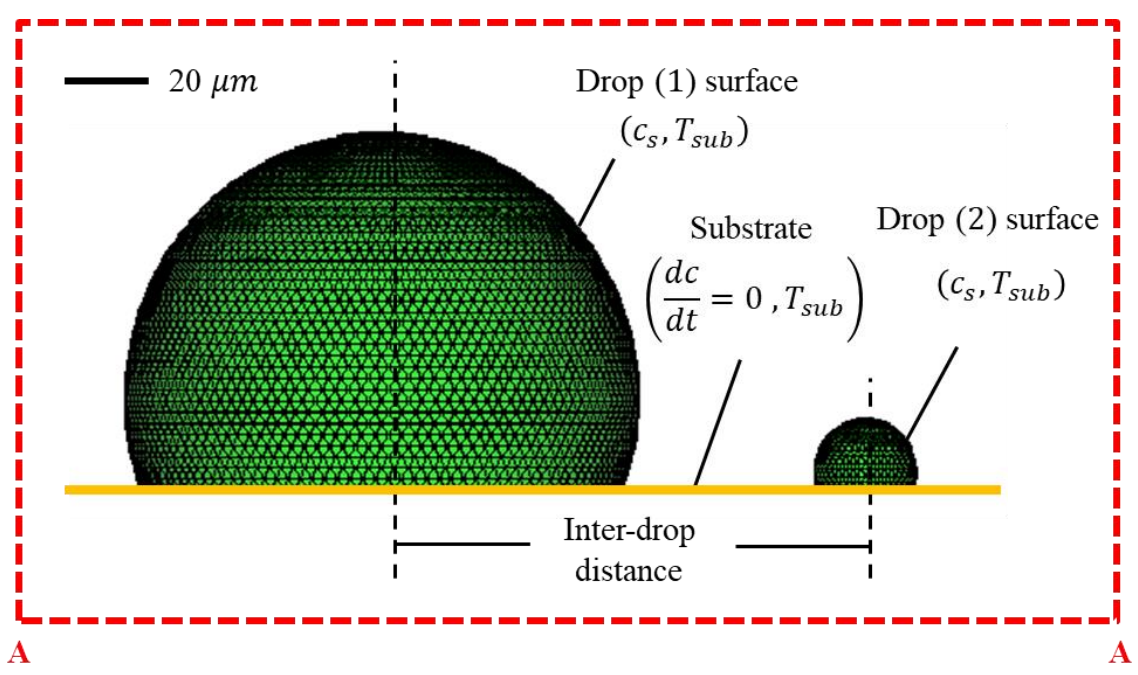

(b)

Figure 2. Schematic diagram of the computational domain and boundary conditions used for numerical simulation of a condensing pair of droplets. (a) The far-field outer boundary of the domain (blue surface) is represented by large spherical cap, with (b) the pair of droplets (green surfaces) located on the substrate at the center (as shown in $\sim 250 \times$ magnified section view A-A). For the case shown in (b), the pair of droplets have contact radii $R_{c 1}=10 \mu \mathrm{m}$ and $R_{c 2}=60 \mu \mathrm{m}$ and are separated at a distance $p=110 \mu \mathrm{m}$. 


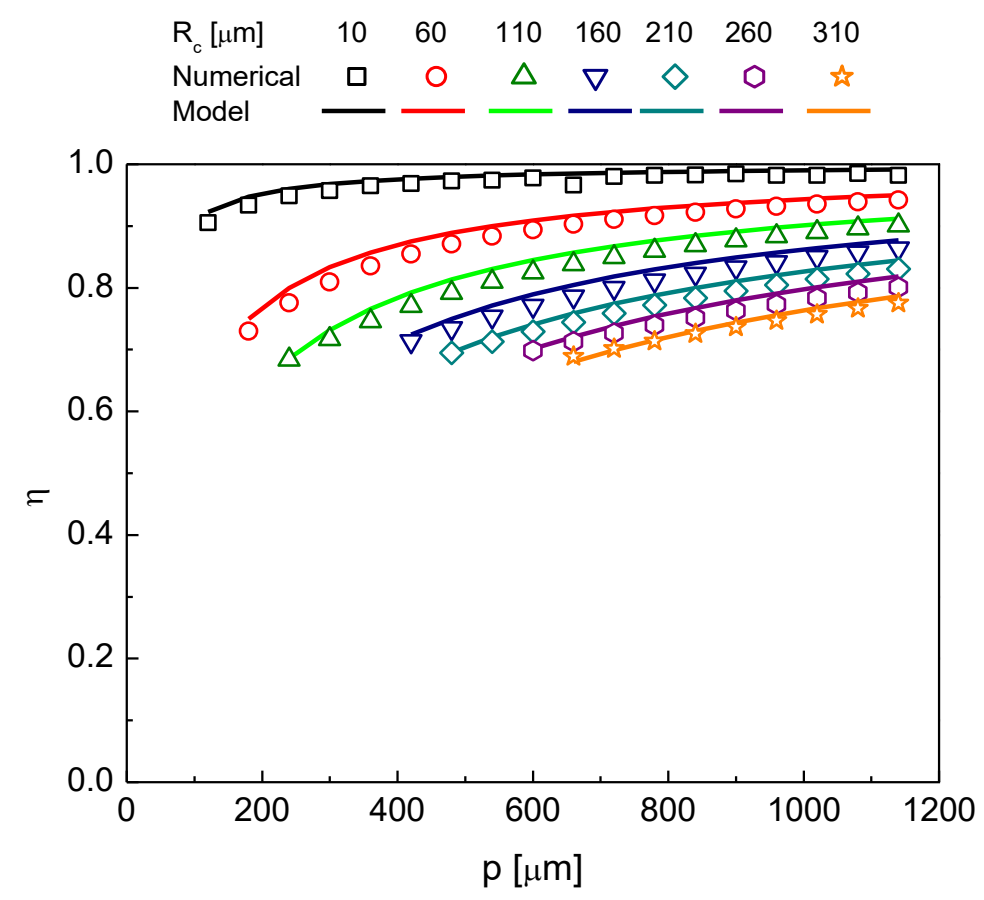

(a)

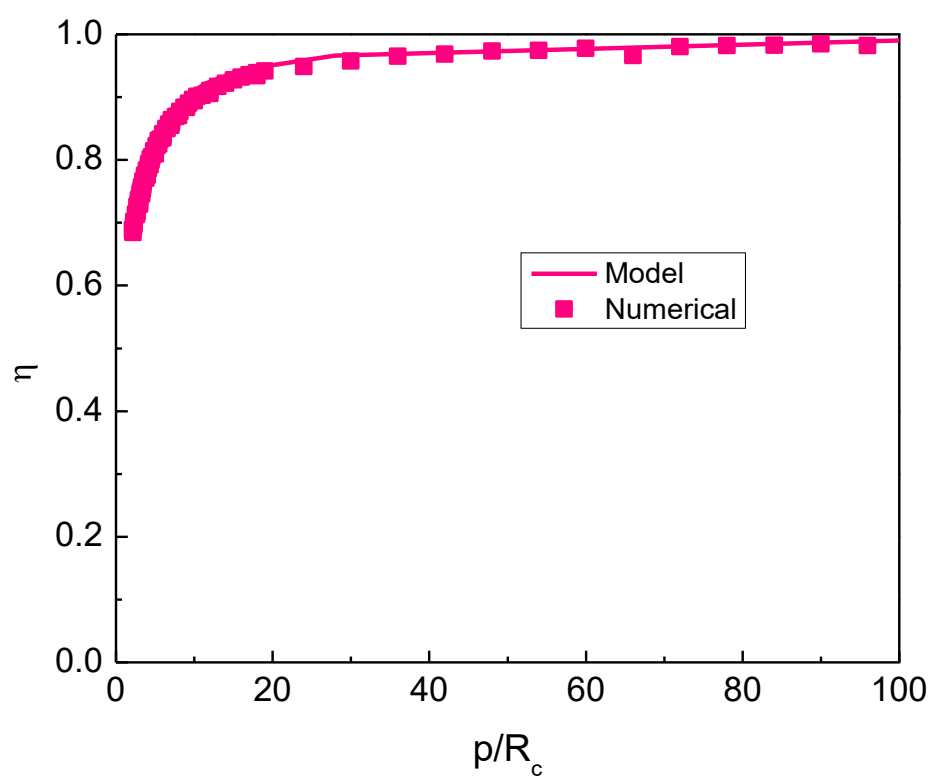

(b)

Figure 3. (a) Condensation rate correction factor $\eta$ for a pair of equally sized droplets having varying contact radii $R_{c}$ as function of droplet pitch $p$ (correction factor compared to the case of an isolated droplet). The predictions using the point sink method are shown as solid lines while results of the numerical simulations are shown as symbols. (b) Correction factor for all of the cases in (a) presented as a function of the ratio between the droplet pitch and contact radii, $p / R_{c}$. 

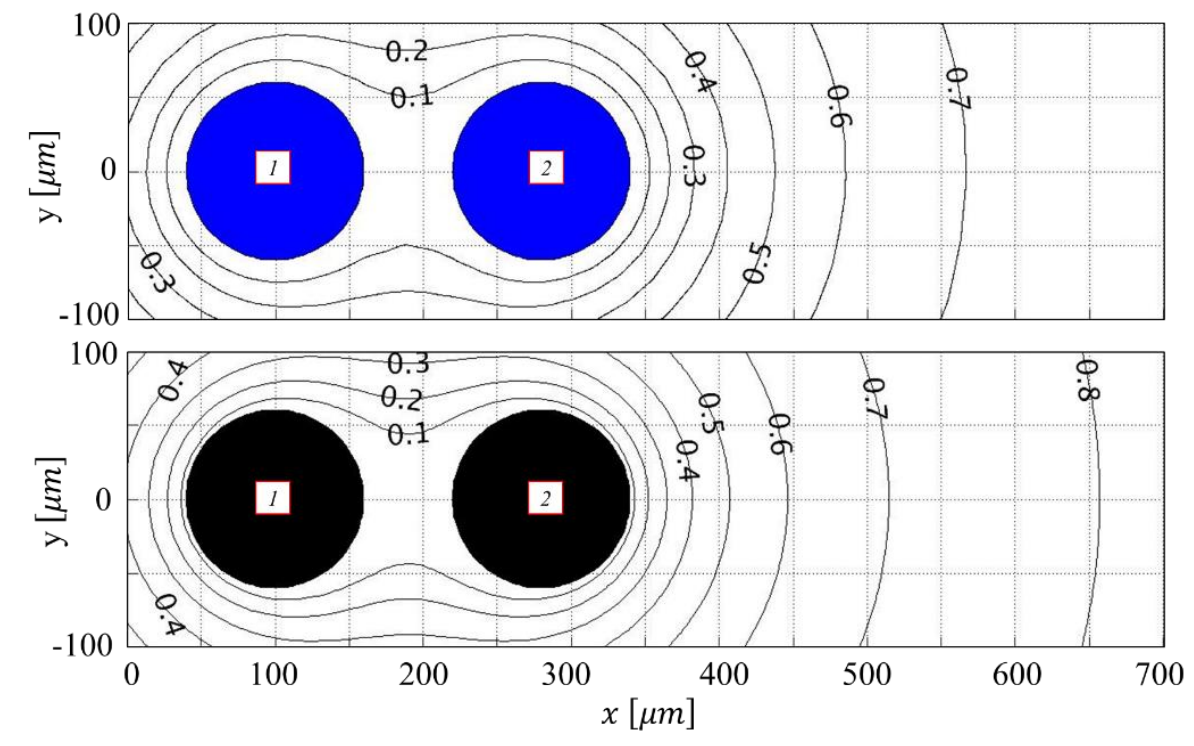

Figure 4. Normalized vapor concentration field $v(\vec{r})$ at the substrate plane in a region surrounding condensing droplets having the same size $R_{c}=R_{c 1}=R_{c 2}=60 \mu \mathrm{m}$ spaced apart at a pitch $p=120 \mu \mathrm{m}$. Results are shown for the numerical simulations (top panel) and for point sink method based prediction (bottom panel). 


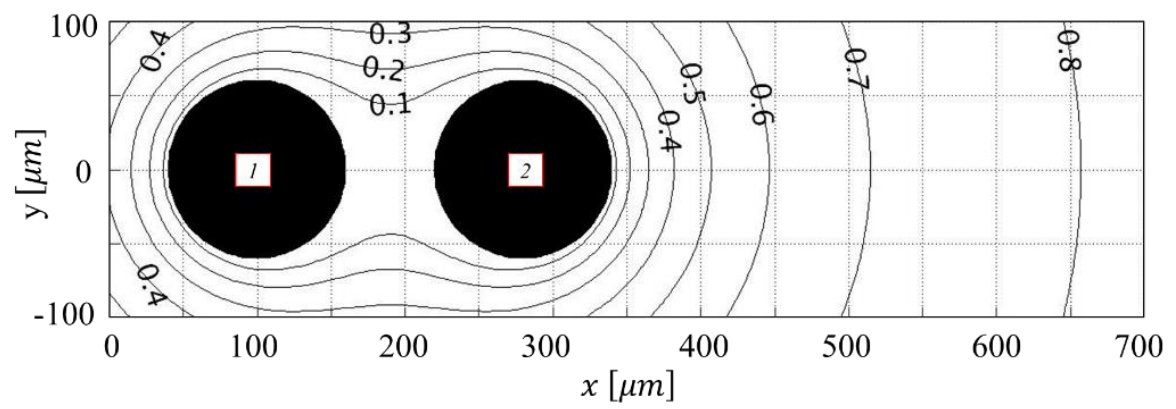

(a)

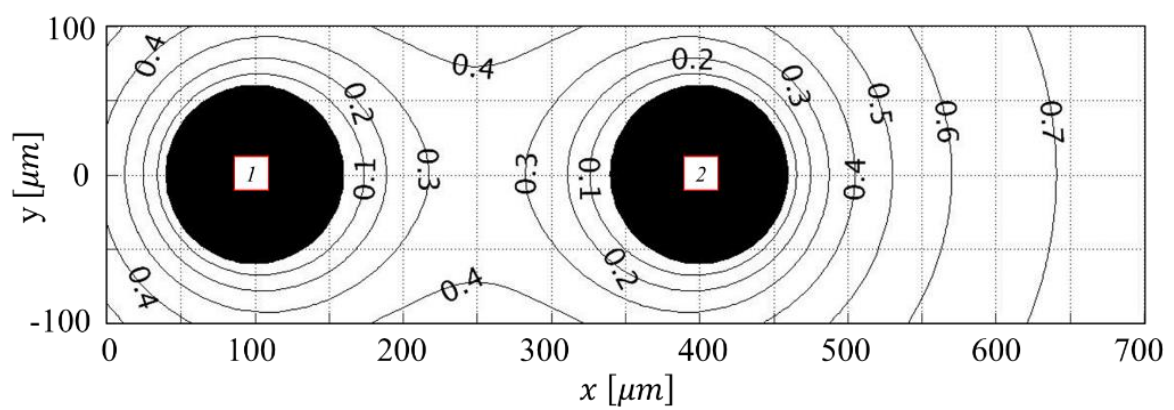

(b)

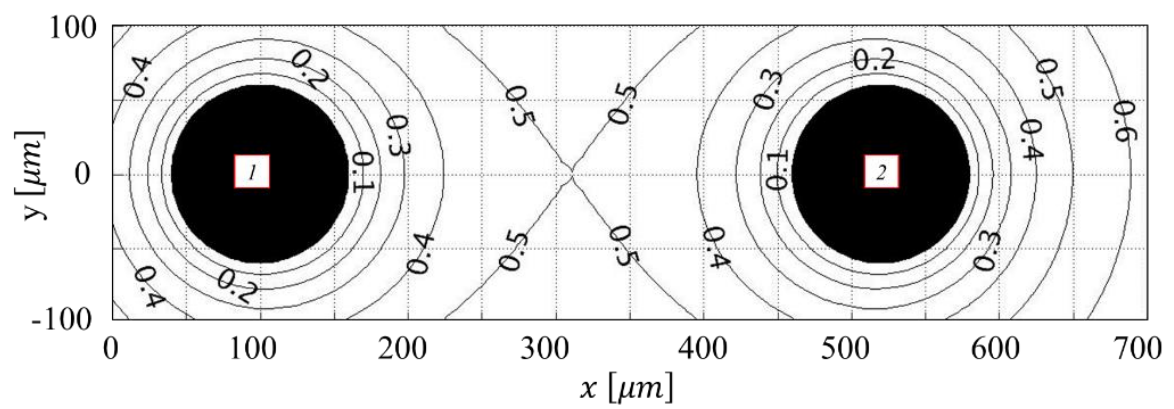

(c)

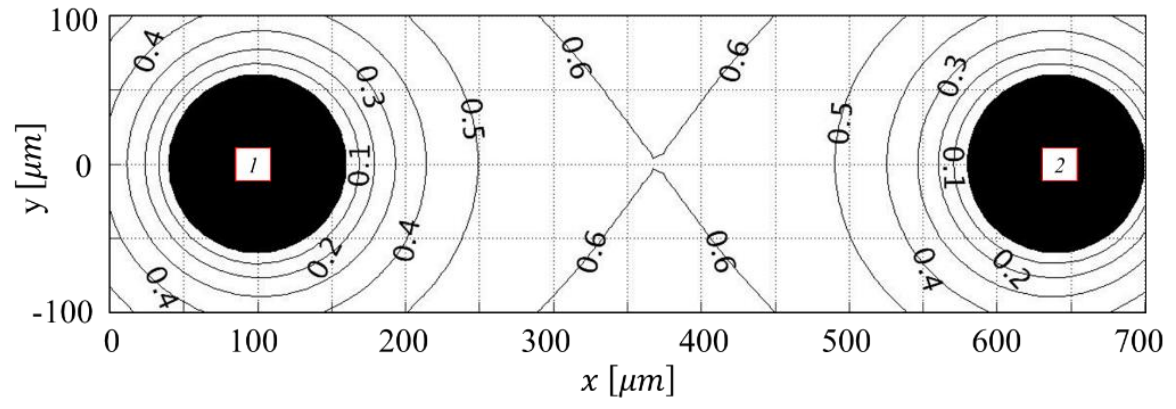

(d)

Figure 5. Normalized vapor concentration field $v(\vec{r})$ at the substrate plane predicted using the point sink superposition method in a region surrounding two condensing droplets having the same size $R_{c}=R_{c 1}=R_{c 2}=60 \mu \mathrm{m}$ and spaced apart at four pitches of (a) $180 \mu \mathrm{m}$, (b) $300 \mu \mathrm{m}$, (c) 420 $\mu \mathrm{m}$, and (d) $540 \mu \mathrm{m}$.. 

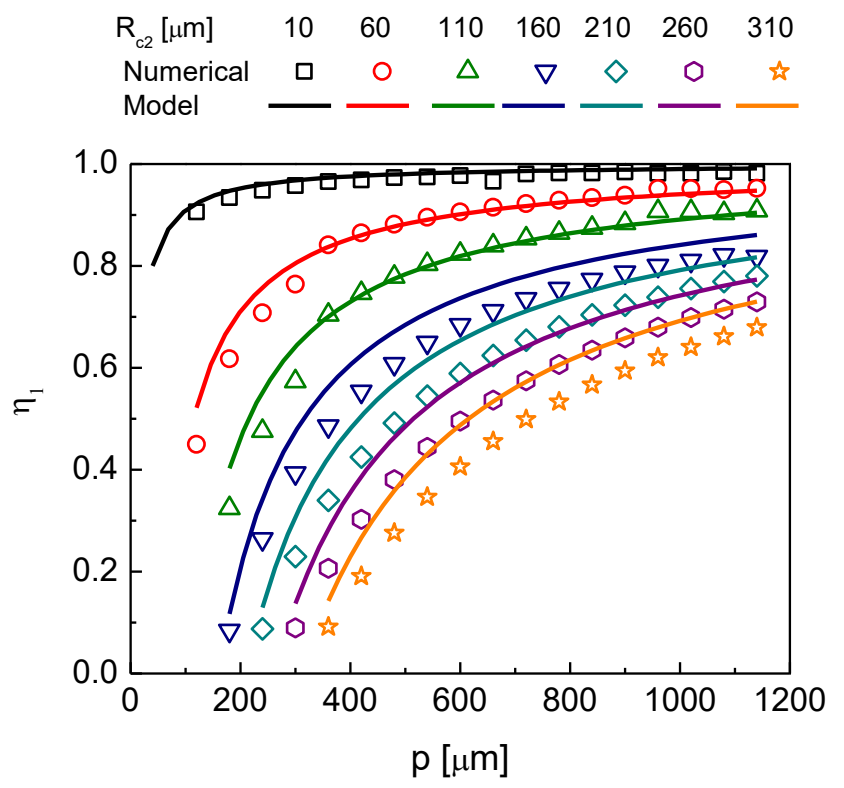

(a)

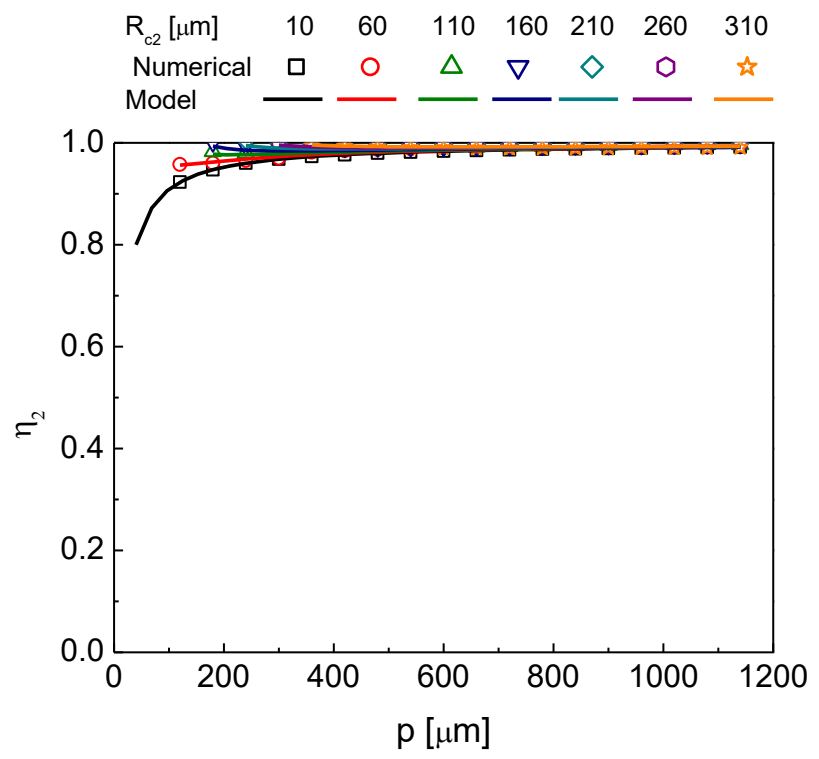

(b)

Figure 6. Condensation rate correction factors (a) $\eta_{1}$ for a small droplet, $R_{c 1}=10 \mu \mathrm{m}$, and (b) $\eta_{2}$ for the relatively larger neighbor droplet, $10 \mu \mathrm{m}<R_{c 2}<310 \mu \mathrm{m}$, as function of the pitch between the two droplets. The predictions using the point sink method are shown as solid lines while results of the numerical simulations are shown as symbols. 


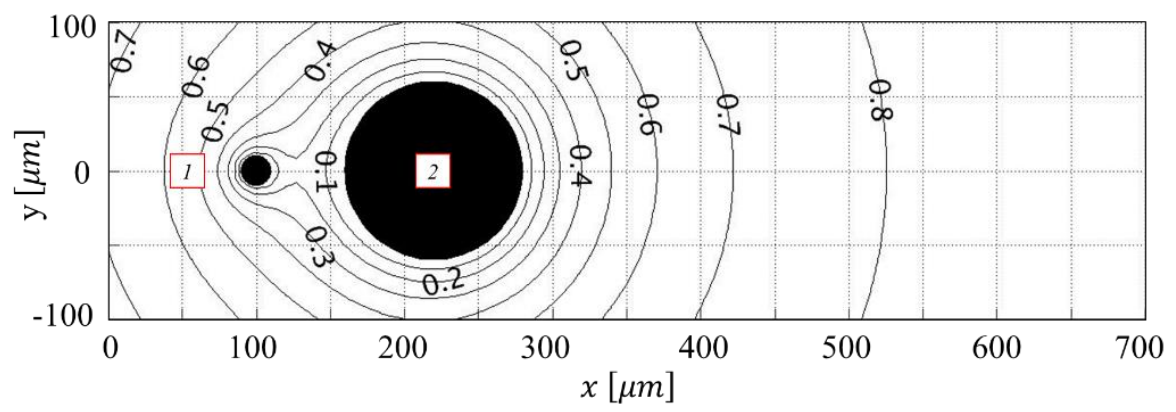

(a)

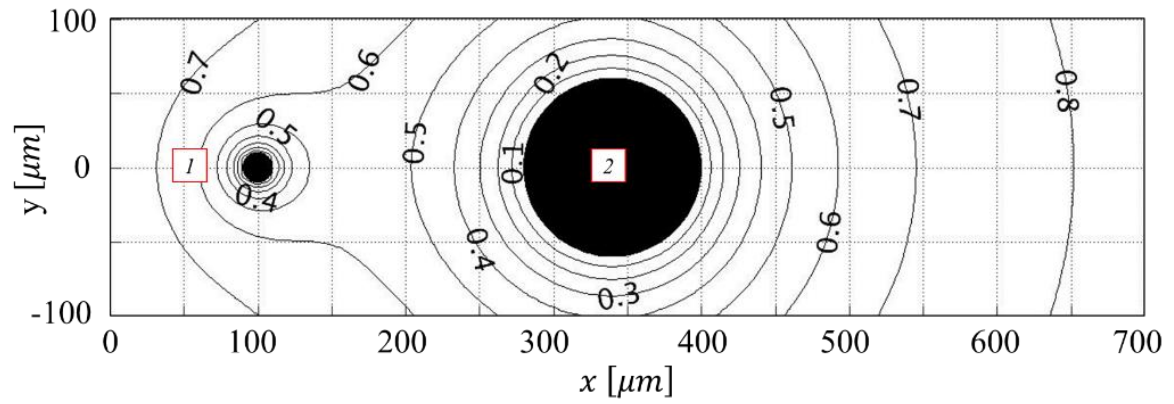

(b)

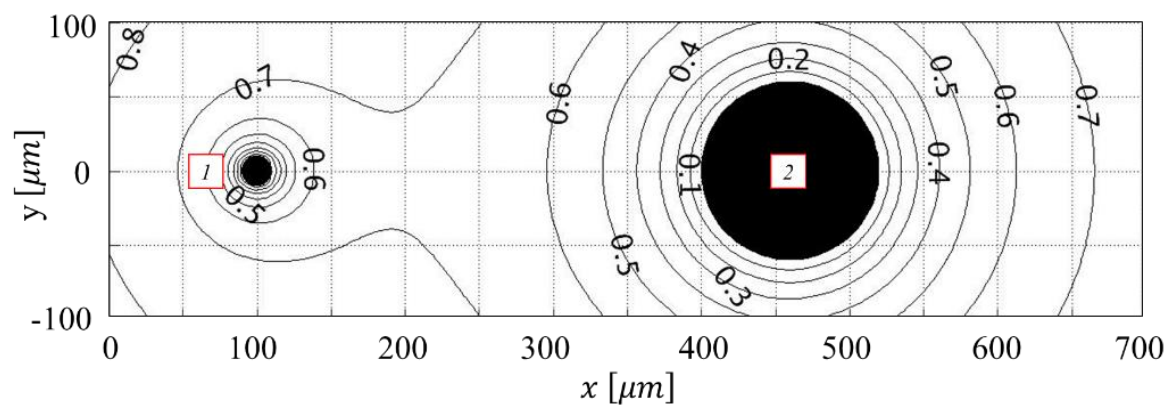

(c)

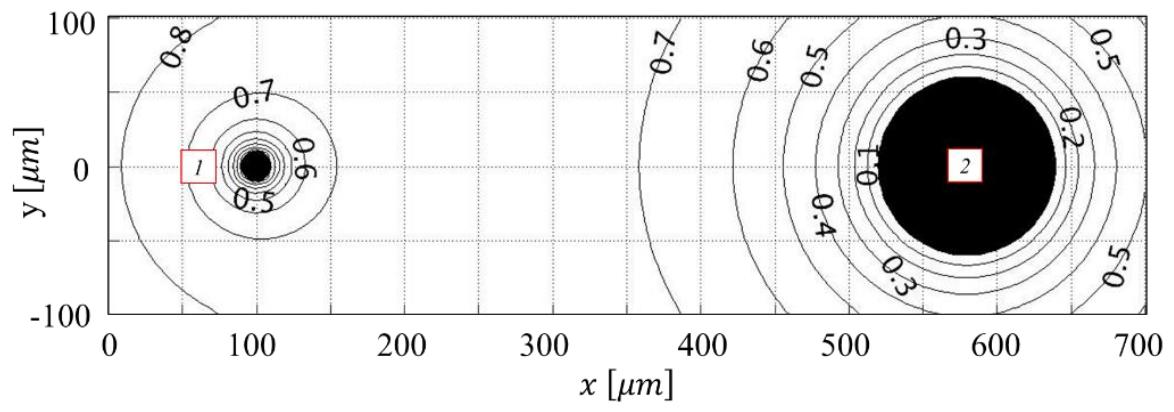

(d)

Figure 7. Normalized vapor concentration field $v(\vec{r})$ at the substrate plane predicted using the point sink superposition method for a small condensing droplet $R_{c 1}=10 \mu \mathrm{m}$ nearby a larger condensing droplet $R_{c 2}=60 \mu \mathrm{m}$ at four pitches of (a) $120 \mu \mathrm{m}$, (b) $240 \mu \mathrm{m}$, (c) $360 \mu \mathrm{m}$, and (d) $480 \mu \mathrm{m}$. 


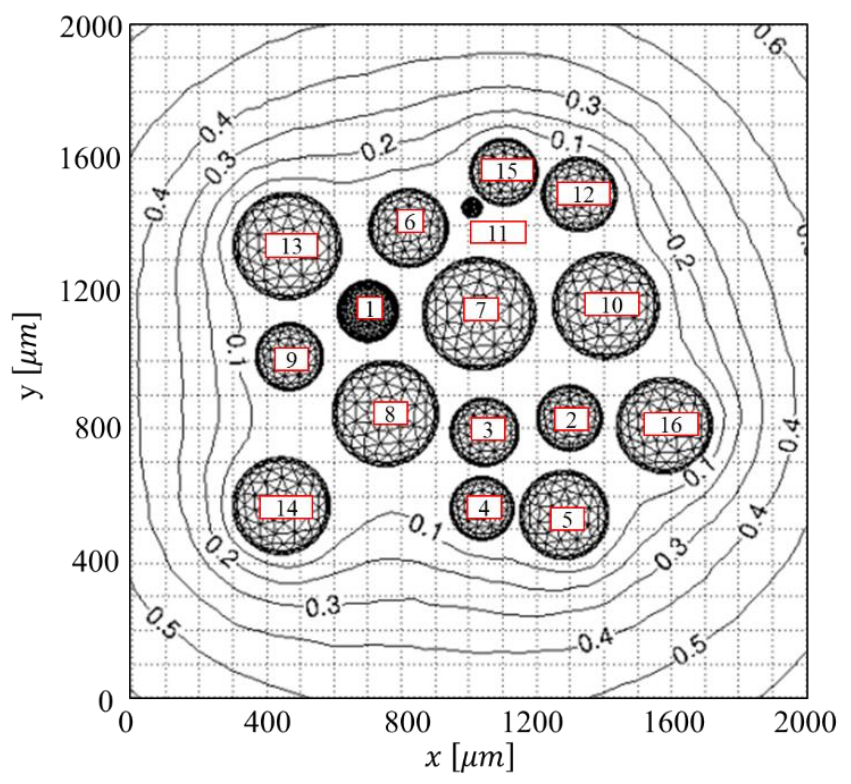

(a)

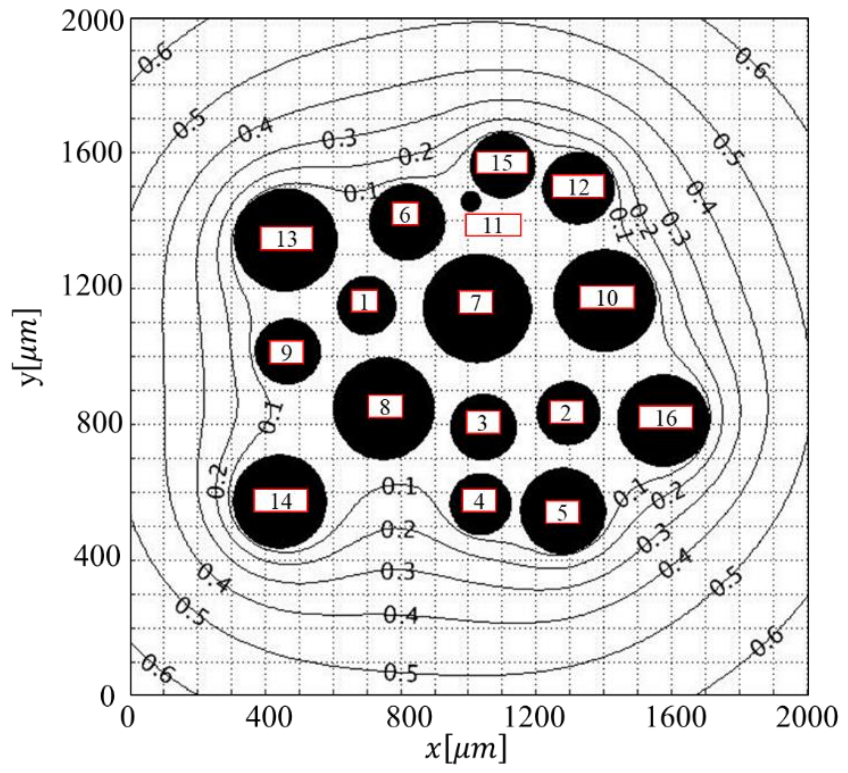

(b)

Figure 8. Normalized vapor concentration field $v(\vec{r})$ at the substrate plane for a system of 16 condensing droplets obtained using (a) the numerical solution of the vapor diffusion equation and (b) the point sink superposition method modeling approach. 


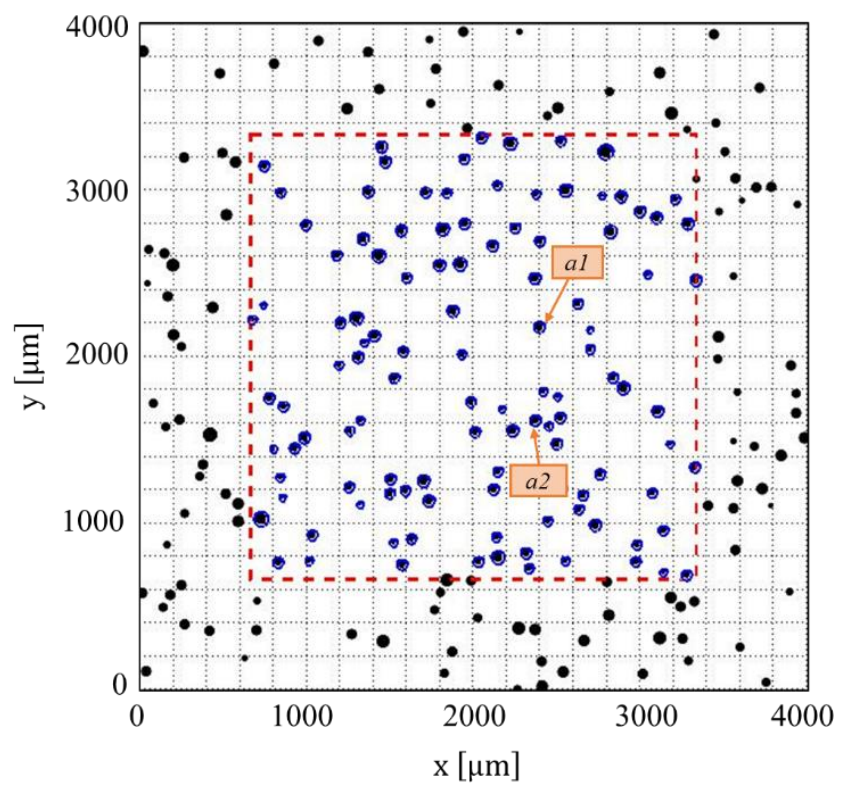

(a)

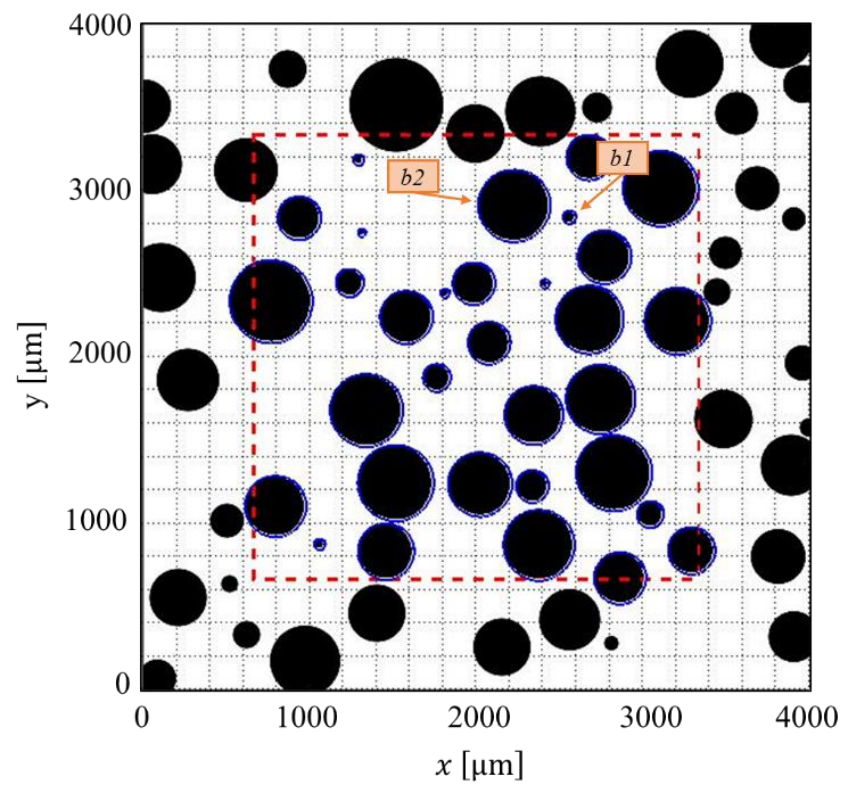

(b)

Figure 9. Systems of randomly generated droplets having (a) 172 droplets with an average radius of $30.1 \mu \mathrm{m}$ and standard deviation of $5.0 \mu \mathrm{m}$ and (b) 66 droplets with average radius of $143.3 \mu \mathrm{m}$ and standard deviation of $69.9 \mu \mathrm{m}$. Analysis of the condensation behavior is restricted to within the dashed squares containing a subset of (a) 112 and (b) 32 droplets. 


\section{Tables}

Table 1. Condensation rate of individual droplets, as well as the overall condensation rate, for the system of droplets shown in Figure 8. The error compares condensation rates from the numerical simulations to those obtained using the point sink superposition method.

\begin{tabular}{cccc}
\hline $\begin{array}{c}\text { Droplet } \\
\text { Tag }\end{array}$ & $\begin{array}{c}\dot{\boldsymbol{m}}\left[\mathbf{x 1 0} \mathbf{x}^{-12} \mathbf{k g} / \mathbf{s}\right] \\
\text { Numerical }\end{array}$ & Model & $\begin{array}{c}\text { Error } \\
{[\%]}\end{array}$ \\
\hline \hline 1 & 3.4 & 3.0 & 11.0 \\
2 & 3.8 & 4.9 & 30.3 \\
3 & 8.5 & 8.6 & 2.1 \\
4 & 19.5 & 21.3 & 9.3 \\
5 & 44.0 & 50.3 & 14.3 \\
6 & 23.6 & 26.5 & 12.3 \\
7 & 17.6 & 27.8 & 58.1 \\
8 & 25.4 & 34.1 & 34.5 \\
9 & 20.6 & 22.6 & 9.7 \\
10 & 42.4 & 50.1 & 18.2 \\
11 & 1.0 & 0.4 & 56.6 \\
12 & 35.3 & 38.7 & 9.7 \\
13 & 70.1 & 79.2 & 13.0 \\
14 & 73.6 & 80.4 & 9.2 \\
15 & 27.9 & 31.2 & 12.1 \\
16 & 59.0 & 65.1 & 10.3 \\
\hline \hline Total & $\mathbf{4 7 5 . 3}$ & $\mathbf{5 4 4 . 2}$ & $\mathbf{1 4 . 5}$ \\
\hline
\end{tabular}


Table 2. (a) Total condensation rates calculated by the point sink method, the isolated droplet growth model, and the filmwise-like growth model for the systems of droplets inside the dashed square shown in Figure 9 (a) and Figure 9 (b). (b) Correction factor for droplets a1, a2, b1 and b2.

\begin{tabular}{cccc}
\hline System & \multicolumn{3}{c}{$\dot{\boldsymbol{m}}\left[\mathbf{x 1 0}^{-10} \mathbf{~ k g} / \mathbf{s}\right]$} \\
& Isolated & Film & Model \\
\hline \hline Figure 9 (a) & 39.2 & 7.9 & 5.1 \\
Figure 9 (b) & 53.3 & 7.9 & 4.7 \\
\hline
\end{tabular}

(a)

\begin{tabular}{cc}
\hline Droplet Tag & $\eta$ \\
\hline \hline$a 1$ & 0.14 \\
$a 2$ & 0.09 \\
$b 1$ & 0.02 \\
$b 2$ & 0.10 \\
\hline
\end{tabular}

(b) 